\title{
CALIDAD DE FRUTO DE LITCHI (Litchi chinensis Sonn.) PRODUCIDO EN EL ESTADO DE VERACRUZ, MÉXICO
}

\author{
FRUIT QUALITY OF LYCHEE (Litchi chinensis Sonn.) PRODUCED \\ IN VERACRUZ STATE, MÉXICO
}

\author{
Aristarco Aguas-Atlahua ${ }^{1}$, Eliseo García-Pérez ${ }^{1 \star}$, Octavio Ruiz-Rosado ${ }^{1}$ y Antonio Trinidad-Santos ${ }^{2}$
}

\begin{abstract}
${ }^{1}$ Campus Veracruz, Colegio de Postgraduados. Km. 88.5 Carr. Federal Xalapa-Veracruz, Predio Tepetates. 91674, Mpio. de Manlio F. Altamirano, Veracruz. Tel. (229) 2010770 y Fax (229) 920 7285. ${ }^{2}$ Campus Montecillo, Colegio de Postgraduados. Km 36.5 Carr. México-Texcoco. 56230, Montecillo, Texcoco, Edo. de México.
\end{abstract}

*Autor para correspondencia (geliseo@colpos.mx; eligarpe05@hotmail.com)

\section{RESUMEN}

Litchi (Litchi chinensis Sonn.) es un fruto originario de China y Vietnam que ya se cultiva en México, donde el principal estado productor es Veracruz con los cultivares 'Mauritius' y 'Brewster', entre otros. Su producción se comercializa en el mercado nacional e internacional. El objetivo de este estudio fue evaluar las características físicas y químicas de los frutos de litchi producidos en las regiones centro y norte del Estado de Veracruz, en seis huertas ubicadas en cinco municipios. Se recolectaron $4 \mathrm{~kg}$ de fruto por huerta y se tomaron al azar 40 frutos para su análisis de calidad. Las variables evaluadas fueron: longitud, diámetro, firmeza, peso del fruto, cáscara y semilla, sólidos solubles totales, acidez titulable y color de cáscara. Los frutos del cultivar 'Mauritius' presentaron variación entre huertas; destacaron los de la huerta Lechuguillas con los frutos más grandes $(35.1 \mathrm{~mm}$ de longitud y $21.2 \mathrm{~g}$ de peso) y mayor peso de pulpa (16.8 g). Además, los frutos de este huerto tuvieron baja acidez, buen color de cáscara, y alto contenido de solidos solubles totales.

Palabras clave: Litchi chinensis, acidez titulable, color de fruto, sólidos solubles totales, tamaño de fruto.

\section{SUMMARY}

Lychee (Litchi chinensis Sonn.) is a fruit originating from China and Vietnam which is cultivated in México also, and Veracruz is cultivated with 'Mauritius' and 'Brewster' cultivars and it is the main state for the national and international markets. The aim of this research was to evaluate the physical and chemical characteristics of lychee fruits produced in six orchards located at the central and northern regions of the state of Veracruz. For this study $4 \mathrm{~kg}$ were sampled per each orchard, from which $\mathbf{4 0}$ fruits were randomly selected for quality evaluation. The variables measured were: length, diameter, firmness, weight of fruit and its components (e.g. pulp, peel and seed), total soluble solids, titratable acidity, and pericarp color. The 'Mauritius' fruits varied in quality amongst orchards. The Lechuguillas orchard showed the biggest fruits with $35.1 \mathrm{~mm}$ length , $21.2 \mathrm{~g}$ of total weight and $16.8 \mathrm{~g}$ of pulp weight. Furthermore, fruits of this site had low acidity, acceptable peel color and high content of total soluble solids.

Index words: Litchi chinensis, titratable acidity, fruit color, total soluble solids, fruit size.

\section{INTRODUCCIÓN}

Litchi (Litchi chinensis Sonn.) es un frutal de clima sub- tropical cuyo centro de origen es el sur de China y norte de Vietnam (Zhou et al., 2008). Se adapta en las zonas tropicales y subtropicales del mundo, con inviernos secos, libres de heladas y humedad relativa alrededor de $75 \%$. En la mayoría de los países donde se cultiva este frutal presenta alternancia de producción determinada por las características genéticas de los cultivares y acentuada por la falta de inviernos frescos, que no permiten una adecuada floración.

Existen evidencias de que temperaturas menores a 20 ${ }^{\circ} \mathrm{C}$ durante un periodo de $45 \mathrm{~d}$ previos a la floración, estimulan la formación de brotes florales lo que favorece la producción de frutos (Mitra y Pathak, 2010). La producción de litchi a nivel mundial es de alrededor de $2.2 \mathrm{mi}$ llones de toneladas (Global Freigth Solutions-US Customs Compliance, 2014); de esta cantidad $95 \%$ se produce en la región de Asia-Pacífico, principalmente en China, India, Tailandia, Australia y Vietnam (Mitra y Pathak, 2010). La producción en el continente americano es menor a $3 \%$ de la producción mundial, y los principales países son Estados Unidos, México y Brasil (Global Freigth Solutions-US Customs Compliance, 2014).

En México el litchi fue introducido a principios del Siglo XX, en el estado de Sinaloa, pero fue hasta las décadas de los 70 y 80 's que se establecieron las primeras plantaciones comerciales, en los estados de Sinaloa, Veracruz y San Luis Potosí (De-la-Garza, 2004). Los cultivares plantados son: 'Brewster,', 'Mauritius', 'Kway My', 'Haak Yip,' 'Groff' y 'Sweet Cliff' (Rinderman y Gómez, 2001). En los últimos diez años las plantaciones de litchi en México han tenido un aumento significativo, y actualmente se reportan 3571 ha a nivel nacional y 1639 ha en el estado de Veracruz.

Los principales estados donde se cultiva litchi son: Veracruz, Oaxaca, Sinaloa, San Luis Potosí, Puebla, Chiapas y Michoacán. Veracruz es el estado con mayor producción, con 8407 t en 2012 (SIAP, 2014); los municipios que destacan son: Papantla, Tihuatlán, Poza Rica, Tuxpan, Martínez 
de la Torre, Tlapacoyan, Paso del Macho, Paso de Ovejas y Córdoba. Una característica de estos municipios es que se encuentran en zonas con climas cálidos, aunque con importantes diferencias en altitud, latitud, precipitación pluvial y suelos, que sin duda generan un comportamiento diferente en la floración, fructificación y en la calidad de los frutos.

La producción de litchi de Veracruz se comercializa a nivel local, regional, nacional e internacional. A nivel nacional, una parte de la producción se lleva a la central de abastos de la ciudad de México, y de allí se distribuye a otras centrales, mercados o supermercados en otras ciudades. En general, la organización de productores es limitada y más aún para la comercialización del fruto en el mercado nacional, lo que ocasiona que los precios de venta sean bajos. El precio medio rural de $1 \mathrm{~kg}$ de fruto fue de 12.89 pesos en el año 2012 (SIAP, 2014). En contraste, existen productores que con buen manejo poscosecha venden a precios de 30 a 40 pesos el kilogramo. Los principales mercados de exportación son los de Estados Unidos, Francia y Canadá, con un precio medio de USD 4.9 (National Agricultural Marketing Council, 2013).

Litchi es un fruto no-climatérico, por lo que su cosecha se realiza cuando alcanza la madurez comercial (Hajare et al., 2010). Presenta un pericarpio delgado (1 a $3 \mathrm{~mm}$ ), de consistencia áspera, textura rugosa y de color rojo intenso o rosa cuando alcanza la madurez de consumo (Pesis et al., 2002); existen frutos de forma esférica, oblonga o con forma de corazón, y miden entre 25 a $40 \mathrm{~mm}$ de longitud (Jiang et al., 2003); presenta un arilo (pulpa) jugoso, con un sabor delicioso (Kumar et al., 2011). El arilo es la parte comestible, que se encuentra cubriendo la semilla; su espesor depende del cultivar y es de color blanco traslucido (Nacif et al., 2001). La semilla representa entre el 10 y $18 \%$ del peso del fruto (Galán-Sauco y Menini, 1987). El fruto está constituido principalmente por agua ( 76 a $87 \%$ del peso de la pulpa), azúcares (11.8 a 20.6\%), y ácidos orgánicos (0.2 a $1.1 \%$ ácido málico) (Carvalho y Salomão, 2000).

Los criterios de calidad para los frutos de litchi se dividen en externos e internos. Los externos incluyen principalmente el color de la cáscara, que es uno de los atributos más importantes en este fruto (Sivakumar y Korsten, 2006). Se prefiere un color rojo brillante; tamaño, peso y forma son otras características importantes; además que esté libre de daños mecánicos, pudriciones y agrietamientos. Los criterios internos incluyen el tamaño de la semilla, los sólidos solubles totales (SST), la acidez titulable (AT) y la relación SST/AT. Estas características presentan variaciones en función del cultivar (Cronje et al., 2009). En México para el cultivar 'Brewster' se reportan SST entre 19.7 a $21.3^{\circ}$ Brix y AT de 0.3 a $0.5 \%$ de ácido málico (Aquino et al., 2010).
A nivel internacional de acuerdo con la Norma CODEX 196, los frutos de litchi para exportación y consumo en fresco se clasifican en extras (con $33 \mathrm{~mm}$ de diámetro ecuatorial), categoría I y categoría II (ambas con $20 \mathrm{~mm}$ de diámetro), además de otras características (FAO/WHO, 2011). La información sobre la calidad de los frutos de litchi que se producen en México es escasa. En el estado de Veracruz no hay evidencias de registros de las características físicas y químicas. De éstas, el tamaño, peso, color, firmeza, contenido de azucares, acidez y sabor son atributos de calidad que juegan un papel importante en la aceptación del consumidor; y son consideradas en el potencial de comercialización en el mercado nacional e internacional. Por tanto, el objetivo del presente trabajo fue determinar las características físicas y químicas de frutos de litchi de los cultivares 'Mauritius' y 'Brewster', colectados en seis diferentes huertas de las regiones centro y norte del estado de Veracruz.

\section{MATERIALES Y MÉTODOS}

La investigación se llevó a cabo de noviembre 2009 a junio de 2010. Se seleccionaron seis huertas, cinco con el cultivar 'Mauritius' y una con 'Brewster' que se ilustran en la Figura 1. Estos cultivares son contrastantes en su ubicación geográfica: latitud, longitud y altitud (Cuadro 1). Las huertas están en las localidades de Tuxpan, Yecuatla, Lechuguillas, Amatlán, El Plan de Manantial y Tolome, en cinco municipios del estado de Veracruz, que tienen los siguientes climas: Aw (tropical con lluvias en verano, subtipos $A_{0} y$ $\mathrm{Aw}_{2}$ ), Af (tropical con lluvias todo el año) y Am (tropical monzónico) (Soto, 1986).

La edad de los árboles varió de 8 a 12 años, y los huertos presentaron diferencias importantes en el manejo agronómico. Las huertas de Tolome, El Plan de Manantial y Lechuguillas, cuentan con un sistema de riego presurizado, en contraste las otras tres huertas que se manejan bajo condiciones de temporal (secano). La huerta de Lechuguillas está certificada como sistema de producción orgánica. Las distancias de plantación en las huertas son de 7 x 7 m y 8 × 8 m.

\section{Toma de muestras y variables medidas}

La colecta de frutos se hizo en los meses de mayo y junio del 2010, cuando los frutos alcanzaron su madurez comercial, identificada por el color rojo de la cáscara en toda la superficie del fruto: tenue para 'Mauritius' e intenso para 'Brewster' (Pesis et al., 2002). En 10 árboles representativos de cada huerta se colectaron $4 \mathrm{~kg}$ de fruto, se transportaron en hieleras al laboratorio y se mantuvieron en refrigeración a $10^{\circ} \mathrm{C}$.

Se tomaron al azar 40 frutos por huerta para medir las variables físicas: Longitud y diámetro del fruto en $\mathrm{mm}$, 


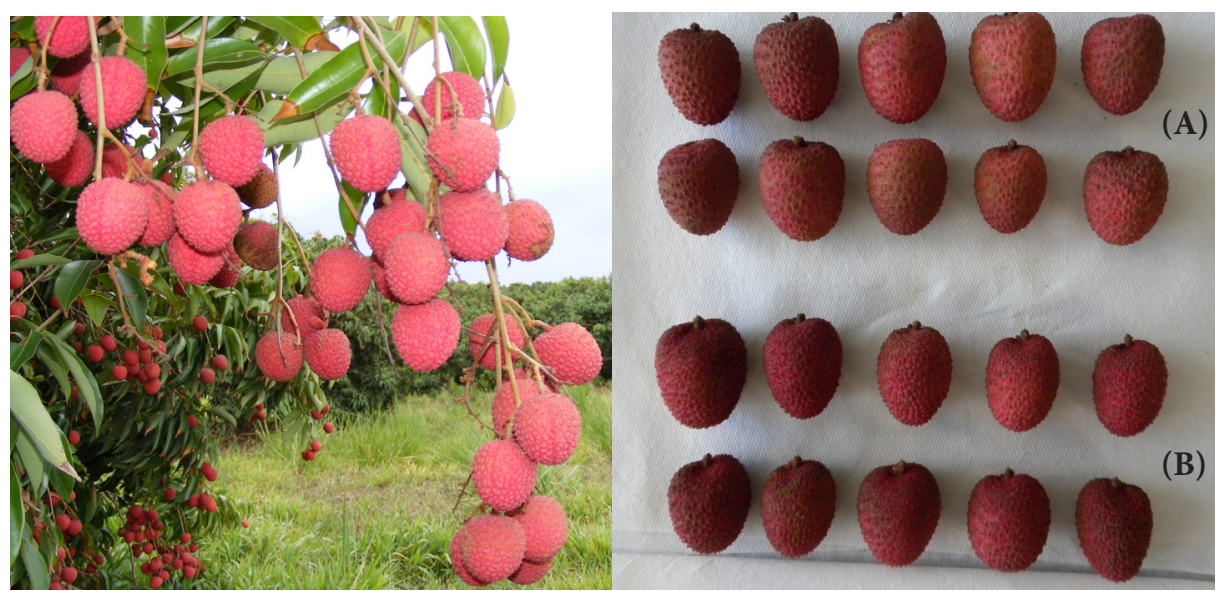

Figura 1. Cultivares de litchi Mauritius(A) y Brewster(B), considerados para el análisis de calidad en el estado de Veracruz.

Cuadro 1. Localización geográfica de huertos de litchi en localidades y municipios en el estado de Veracruz, México, 2010.

\begin{tabular}{lcccc}
\hline Huertas & Cultivar & Latitud Norte & Longitud Oeste9 & Altitud $(\mathrm{m})^{9}$ \\
\hline Tuxpan & 'Mauritius' & $20^{\circ} 54^{\prime} 58.4^{\prime}$ & $97^{\circ} 25^{\prime} 29.2^{\prime}$ & 7 \\
Yecuatla & 'Mauritius' & $19^{\circ} 53^{\prime} 23^{\prime}$ & $96^{\circ} 45^{\prime} 17.6^{\prime}$ & 309 \\
Lechuguillas & 'Mauritius' & $19^{\circ} 59^{\prime} 40.6^{\prime}$ & $96^{\circ} 35^{\prime} 12.9^{\prime}$ & 15 \\
Amatlán & 'Mauritius' & $18^{\circ} 50^{\prime} 47.7^{\prime}$ & $96^{\circ} 23^{\prime} 08.9^{\prime}$ & 732 \\
El Plan de Manantial & 'Mauritius' & $19^{\circ} 12^{\prime} 57.81^{\prime}$ & $96^{\circ} 22^{\prime} 36.7^{\prime}$ & 28 \\
Tolome & 'Brewster' & $19^{\circ} 16^{\prime} 18.9^{\prime}$ & $96^{\circ} 24^{\prime} 14.2^{\prime}$ & 29 \\
\hline
\end{tabular}

'Datos obtenidos con GPS Garmin (map76CSx®, Taiwan).

medidos de la base al ápice y en la parte media del fruto, con un vernier digital de $150 \mathrm{~mm}$ (Mituyo ${ }^{\circledR}$; Kawasaki, Japón). Peso de fruto, cáscara y semilla, en gramos (g), que se registró con una balanza digital ES-3000H (JD3000®; USA). Peso de pulpa (g), que se obtuvo por diferencia entre el peso total de fruto menos el peso de la cáscara y de la semilla. Firmeza del fruto en newtons $(\mathrm{N})$, que se determinó con un penetrómetro digital (FM02380®; Oberdischingen, Germany), provisto con un punzón de $5 \mathrm{~mm}$ de diámetro, $\mathrm{y}$ se realizaron dos punciones en la parte ecuatorial del fruto con cáscara.

El color de fruto (ángulo de matiz y cromaticidad) se determinó con un colorímetro triestímulo (Angguang WSD$3 \mathrm{~A}($; Beijing, China). El equipo se calibró con una placa de porcelana blanca, luego se tomaron las lecturas en la parte media de cada fruto y se registraron los valores X, Y, y Z, y posteriormente se hizo la conversión $a L^{*}, a^{*} y b^{*}$ conforme a la metodología de Wyszecki y Stiles (1982). El sistema de medición de la Comisión Internacional en Iluminación determinó que $\mathrm{L}^{\star}$ mide la luminosidad de la superficie, $\mathrm{a}^{\star}$ representa el color verde o rojo, $\mathrm{y} \mathrm{b}^{*}$ el color azul o amari- llo (Aular et al., 2002). Lo anterior con el fin de calcular el ángulo de matiz $\left({ }^{\circ} \mathrm{Hue}\right.$ ) y la cromaticidad $\left(\mathrm{C}^{\star}\right)$. Los ${ }^{\circ} \mathrm{Hue}$ varían de $0^{\circ}$ a $360^{\circ}\left(0^{\circ}=\right.$ rojo, $90^{\circ}=$ amarillo, $180^{\circ}=$ verde $y$ $270^{\circ}=$ azul) (McGuire, 1992). La pureza de color o cromaticidad $\left(C^{\star}\right)$ expresa la proporción del contenido cromático.

Las ecuaciones aplicadas fueron ${ }^{\circ} \mathrm{Hue}=\tan ^{-1}\left(b^{*} / a^{*}\right)$ y $C^{*}=\left(a^{* 2}+b^{* 2}\right)^{0.5}$ (López y Gómez, 2004). En las variables químicas: sólidos solubles totales, se determinaron con un refractómetro digital (ATAGO PAL-1®; Tokyo, Japón) (Intervalo 0 - $32^{\circ} \mathrm{Brix}$ ), previamente calibrado con una gota de agua destilada; después se colocó una gota de jugo de litchi de cada fruto en el refractómetro y se tomó la lectura.

Para la acidez titulable (\% ácido málico), el jugo de litchi se extrajo de forma manual de cada fruto. Se colocaron 5 $\mathrm{mL}$ de jugo en un vaso de precipitados de $250 \mathrm{~mL}$ y dos gotas del indicador fenolftaleína. Para hacer la titulación se utilizó hidróxido de sodio al $0.1 \mathrm{~N}$. La acidez total se calculó en porcentaje de ácido málico, de acuerdo con la técnica volumétrica descrita por la AOAC (2000). 


\section{Análisis de datos}

El análisis estadístico de los datos, se hizo con el programa SAS ${ }^{\circ}$ v. 9.3 para Windows (SAS Institute, 2004), mediante análisis de varianza bajo el diseño completamente al azar, con el procedimiento PROC ANOVA y prueba de medias (Tukey, 0.05), con los datos de las cinco huertas para el cultivar 'Mauritius'. Para 'Brewster' solamente se presentan las medias y desviaciones estándar de las características físicas de los frutos, como referencia entre cultivares.

\section{RESULTADOS Y DISCUSIÓN}

Los climas en las diferentes localidades de las huertas se muestran en el Cuadro 2, que incluyen las medias de las temperaturas máxima, media y mínima de los meses de noviembre y diciembre de 2009 y enero de 2010, que corresponden al periodo de floración y al cuajado inicial de frutos. Las temperaturas luego se incrementaron durante los meses de desarrollo del fruto (febrero-mayo), condición necesaria según Galán-Sauco y Menini (1987), pero las diferencias entre huertas persisten presumiblemente determinadas por el tipo de clima en cada localidad y por los suelos de diferente textura.

Entre las cinco huertas plantadas con el cultivar 'Mauritius', se encontraron diferencias significativas en todas las variables evaluadas, de acuerdo con la prueba de Tukey $(\mathrm{P} \leq 0.05)$. Los frutos de la huerta de Lechuguillas fueron superiores a los de las otras huertas en cuatro variables: longitud, diámetro, peso total de fruto y peso de pulpa. Esto se asocia con el hecho de que esta huerta cuenta con riego presurizado y cuenta con sistema de manejo orgánico, además de tener suelo franco.

Para la variable peso de cáscara las huertas de Amatlán y El Plan de Manantial fueron similares entre ellas pero superiores a las otras tres huertas; las características de manejo de estas huertas, sin riego e insumos para Amatlán y riego errático y pocos insumos en el Plan de Manantial, al parecer no afectan el peso de la cáscara, pero sí al peso de pulpa y fruto por la menor disponibilidad de agua y nutrientes. Los frutos de la huerta de Tuxpan presentaron el menor peso de semilla con $1.7 \mathrm{~g}$, y también fueron los frutos más pequeños junto con los de Yecuatla; ambas huertas carecen de sistema riego, lo que hace suponer que la falta de agua afecta al crecimiento del fruto y sus componentes, aun en condiciones climáticas favorables para el cultivo (Cuadro 3).

Los frutos del cultivar 'Brewster' establecidos en la huerta de Tolome desarrollaron una longitud de $36.3 \mathrm{~mm}$, diámetro $28.8 \mathrm{~mm}$, peso $19.3 \mathrm{~g}$, cáscara $4.1 \mathrm{~g}$, pulpa $11.9 \mathrm{~g}$ y peso de semilla de $3.0 \mathrm{~g}$. Estos frutos de 'Brewster' son de mayor longitud y peso de cáscara y semilla, pero menor diámetro, peso de fruto y de pulpa que los frutos del cultivar 'Mauritius', lo que puede deberse a diferencias entre cultivares o a diferencias ambientales.

Los valores de tales variables fueron inferiores a los reportados por Galán-Sauco y Menini (1987) y Menzel (2002), quienes señalaron un tamaño de hasta $50 \mathrm{~mm}$ de longitud y $40 \mathrm{~mm}$ de diámetro y peso de $22 \mathrm{a} 26 \mathrm{~g}$ para el cultivar 'Mauritius' y 20 a 26 g para 'Brewster'. En un estudio reciente hecho en México con el cultivar 'Mauritius' se reportó un peso medio de fruto de 21 a $25 \mathrm{~g}$ y un diámetro de 30 a 40 $\mathrm{mm}$ (Aquino et al., 2010). Los únicos frutos que están entre los intervalos citados anteriormente, son los de la huerta Lechuguillas que cuenta con el mejor manejo agronómico del cultivo.

Otros estudios realizados en África con el cultivar 'Mauritius' reportan frutos con un peso medio de $23.8 \mathrm{~g}$ (DeReuck et al., 2009) y en Sudáfrica de 18 a $22 \mathrm{~g}$ (Cronje et al., 2009). Estas variaciones se pueden relacionar con las condiciones edafo-climáticas, como se constató en las huertas evaluadas en este estudio (Cuadro 2), y con el manejo agronómico de los árboles. El diámetro ecuatorial de los frutos en las seis huertas aquí evaluadas alcanzó el calibre mínimo

Cuadro 2. Climas y temperaturas medias (Nov. y Dic. 2009, Ene. 2010) en diferentes huertas de litchi en el estado de Veracruz.

\begin{tabular}{lcccccc}
\hline Huertas & Cultivar & Clima & $\operatorname{Tmáx}\left({ }^{\circ} \mathrm{C}\right)^{g}$ & $\operatorname{Tmedia}\left({ }^{\circ} \mathrm{C}\right)$ & $\operatorname{Tmín}\left({ }^{\circ} \mathrm{C}\right)^{g}$ & $\begin{array}{c}\text { Textura de } \\
\text { suelo }\end{array}$ \\
\hline Tuxpan & 'Mauritius' & $\mathrm{Aw}_{2}{ }^{\prime}(\mathrm{e})$ & 24.24 & 19.66 & 15.08 & Franco arcilloso \\
Yecuatla & 'Mauritius' & $\mathrm{Af}(\mathrm{m})(\mathrm{e})$ & 24.99 & 21.09 & 17.19 & Arcilla \\
Lechuguillas & 'Mauritius' & $\mathrm{Aw}_{2}{ }^{\prime}(\mathrm{w})(\mathrm{i})$ & 25.15 & 20.33 & 15.51 & Franco \\
Amatlán & 'Mauritius' & $\mathrm{Am}(\mathrm{i}) \mathrm{g}$ & 28.44 & 20.86 & 13.56 & Arcilla \\
El Plan de Manantial & 'Mauritius' & $\mathrm{Aw}_{0}{ }^{\prime}(\mathrm{w})(\mathrm{i}) \mathrm{g}$ & 27.40 & 22.77 & 18.10 & Franco \\
Tolome & 'Brewster' & $\mathrm{Aw}_{0}{ }^{\prime}(\mathrm{w})(\mathrm{i}) \mathrm{g}$ & 29.15 & 22.96 & 16.76 & Franco arcilloso \\
\hline
\end{tabular}

$\mathrm{Aw}=$ tropical con lluvias en verano, subtipos $\mathrm{Aw}_{0} \mathrm{y} \mathrm{Aw}_{2}, \mathrm{Af}=$ tropical con lluvias todo el año; $\mathrm{Am}=$ tropical monzónico (Soto, 1986). ${ }^{\circ} \mathrm{Temperaturas} \mathrm{obtenidas}$ con termómetros de máximas y mínimas TFA®. 
Cuadro 3. Características físicas del fruto de litchi de los cultivares 'Mauritius' y 'Brewster' en el estado de Veracruz, cosecha 2010.

\begin{tabular}{|c|c|c|c|c|c|c|}
\hline & \multirow{2}{*}{ Longitud (mm) } & \multirow{2}{*}{ Diámetro $(\mathrm{mm})$} & \multicolumn{4}{|c|}{ Peso (g) } \\
\hline & & & Fruto & Cáscara & Pulpa & Semilla \\
\hline \multicolumn{7}{|c|}{ 'Mauritius' (Racimo rojo) } \\
\hline Yecuatla & $33.0 \pm 1.7 \mathrm{c}$ & $28.4 \pm 1.5 \mathrm{c}$ & $17.3 \pm 2.3 \mathrm{c}$ & $2.0 \pm 0.6 \mathrm{~b}$ & $12.9 \pm 2.2 \mathrm{c}$ & $2.3 \pm 1.4 \mathrm{ab}$ \\
\hline Lechuguillas & $35.1 \pm 1.7 \mathrm{a}$ & $30.9 \pm 1.2 \mathrm{a}$ & $21.2 \pm 2.3 \mathrm{a}$ & $1.9 \pm 0.6 \mathrm{bc}$ & $16.8 \pm 1.7 \mathrm{a}$ & $2.5 \pm 0.7 \mathrm{a}$ \\
\hline El Plan de Manantial & $34.0 \pm 2.1 \mathrm{bc}$ & $28.2 \pm 1.7 c$ & $15.3 \pm 2.7 \mathrm{~d}$ & $2.4 \pm 0.4 \mathrm{a}$ & $10.3 \pm 2.0 \mathrm{~d}$ & $2.5 \pm 0.5 \mathrm{a}$ \\
\hline DMS & 1.12 & 0.89 & 1.5 & 0.3 & 1.2 & 0.6 \\
\hline \multicolumn{7}{|c|}{ 'Brewster' (Ralo rojo) } \\
\hline Tolome & $36.3 \pm 1.4$ & $28.8 \pm 2.5$ & $19.1 \pm 2.2$ & $4.1 \pm 0.6$ & $11.9 \pm 2.7$ & $3.0 \pm 1.0$ \\
\hline
\end{tabular}

'Media y desviación estándar de 40 frutos para cada huerta. ${ }^{9}$ Letras iguales de una misma columna no son estadísticamente diferentes $($ Tukey, 0.05$)$. DMS = diferencia mínima significativa.

de $20 \mathrm{~mm}$, que corresponde a las categorías I y II de la norma de calidad del Código Alimentario Internacional (FAO/ WHO, 2011) para la exportación de frutas.

Las huertas con el cultivar 'Mauritius' presentaron diferencias significativas $(P \leq 0.05)$ en la variable firmeza de fruto, ya que las huertas de Yecuatla y El Plan de Manantial tuvieron los valores más altos $(41.7$ y $43.2 \mathrm{~N})$ y superaron al resto de las huertas (Cuadro 4). El cultivar 'Brewster' presentó también una alta firmeza con $44.3 \mathrm{~N}$. La firmeza del fruto en 'Mauritius', en general es inferior al intervalo de 45 a $48 \mathrm{~N}$ reportado por Sivakumar y Korsten (2006), lo cual indica que es necesario mejorar el manejo de las huertas para obtener frutos más firmes.

En acidez titulable (AT) los frutos de 'Mauritius' de la huerta de Tuxpan presentaron la menor media con $0.3 \%$, en contraste con los de El Plan de Manantial que tuvo la mayor con 0.9 \% (Cuadro 4). En sólidos solubles totales (SST) el valor más alto $\left(20.4{ }^{\circ} \mathrm{Brix}\right)$ fue para los frutos de la huerta Yecuatla. En los frutos de ambos cultivares los valores de SST fluctuaron entre 18.26 a $20.48^{\circ} \mathrm{Brix}$, y los de AT variaron de 0.3 a $0.9 \%$. Estos valores están dentro de los intervalos reportados por Galán-Sauco y Menini, (1987) y Carvalho y Salomão (2000), con 11.8 a $20.6{ }^{\circ}$ Brix y de 0.2 a $1.1 \%$ de ácido málico para los cultivares 'Mauritius' y 'Brewster'. Datos similares fueron reportados para frutos de litchi de los cultivares 'Muzaffarpur', 'Heung Lai' y 'Brewster' (Neog y Saikia, 2001).

En color de fruto, con un ángulo ${ }^{\circ} \mathrm{Hue}$ de $46.4^{\circ}$ y 37.0 de cromaticidad la huerta de Amatlán fue superior a las otras huertas, debido a que el color de la cáscara fue menos intenso, lo que demerita la calidad del fruto: esto tiene relación con la ubicación geográfica y el escaso manejo agronómico en este huerto. El color de los frutos de las huertas de Tuxpan, Yecuatla y Lechuguillas, cuyos ángulos ${ }^{\circ} \mathrm{Hue}$ fueron de 39.8 a $42.0^{\circ}$ y su cromaticidad de 32.4 a 35.6 , es cercano a los reportados por Sivakumar y Korsten (2006) para el cultivar 'Mauritius' con $39.7^{\circ}$ Hue y 30.3 de cromaticidad, que representan un color rosa-rojizo. Estos resultados sugieren que en esas localidades existen mejores condiciones edafoclimáticas para el cultivo de litchi.

Las variaciones encontradas en los frutos del cultivar 'Mauritius' se podrían atribuir al efecto de factores ambientales, como la temperatura que tiene una influencia importante (Menzel, 2002) en el litchi. Así, las bajas temperaturas en otoño-invierno favorecen la floración, y las temperaturas altas en primavera permiten mejor crecimiento y desarrollo de frutos (Cuadro 2). El suelo es otro factor importante, cuya textura varió de arcillosa a franca en las huertas, lo que representa importantes cambios en contenido de materia orgánica y nutrientes, capacidad de retención de humedad, $\mathrm{pH}$ y otras propiedades. Además, las diferencias en el manejo agronómico, como es el caso de la huerta de Lechuguillas que cuenta con manejo orgánico. Al respecto, Pesis et al. (2002) indicaron que en un mismo cultivar la composición química de los frutos de litchi pueden presentar variabilidad por influencia de las condiciones climáticas. Por ello Valle-Guadarrama et al. (2008) sugirieron el establecimiento de normas de calidad de los frutos de litchi, específicas para cada región productora.

\section{Componentes del fruto}

El arilo que es la parte comestible del fruto de litchi, en 'Mauritius' su peso fue de 67.4 a $79.1 \%$ del peso del fruto y en 'Brewster' de $62.3 \%$. La cáscara osciló de 8.9 a $15.9 \%$ del peso de fruto en 'Mauritius' y en 'Brewster' representó 
Cuadro 4. Parámetros de calidad en el fruto de litchi de los cultivares 'Mauritius' y 'Brewster' en el estado de Veracruz, cosecha 2010.

\begin{tabular}{|c|c|c|c|c|c|}
\hline Huertas & Firmeza $(\mathrm{N})$ & AT (\%) & SST $\left({ }^{\circ}\right.$ Brix $)$ & ${ }^{\circ} \mathrm{Hue}$ & Chroma \\
\hline \multicolumn{6}{|c|}{ 'Mauritius' (Racimo rojo) } \\
\hline Tuxpan & $37.6 \pm 5.6^{9} b^{99}$ & $0.3 \pm 0.6 \mathrm{e}$ & $18.2 \pm 1.5 \mathrm{c}$ & $39.8 \pm 6.6 \mathrm{c}$ & $32.4 \pm 3.0 \mathrm{~d}$ \\
\hline Yecuatla & $41.7 \pm 5.0 \mathrm{a}$ & $0.8 \pm 0.1 \mathrm{c}$ & $20.4 \pm 1.3 \mathrm{a}$ & $40.0 \pm 8.7 \mathrm{c}$ & $35.6 \pm 1.6 \mathrm{ab}$ \\
\hline Lechuguillas & $35.0 \pm 4.5 \mathrm{~b}$ & $0.4 \pm 0.7 \mathrm{~d}$ & $19.3 \pm 1.3 \mathrm{~b}$ & $42.0 \pm 6.1 \mathrm{~b}$ & $33.7 \pm 3.0 \mathrm{~cd}$ \\
\hline Amatlán & $34.8 \pm 3.0 \mathrm{~b}$ & $0.9 \pm 0.1 \mathrm{~b}$ & $19.4 \pm 0.9 \mathrm{~b}$ & $46.4 \pm 4.6 \mathrm{a}$ & $37.0 \pm 1.8 \mathrm{a}$ \\
\hline El Plan de Manantial & $43.2 \pm 6.0 \mathrm{a}$ & $0.9 \pm 0.1 \mathrm{a}$ & $19.9 \pm 1.1 \mathrm{ab}$ & $38.7 \pm 5.1 \mathrm{c}$ & $34.4 \pm 2.2 \mathrm{bc}$ \\
\hline DMS & 3.07 & 0.06 & 0.77 & 3.93 & 1.50 \\
\hline \multicolumn{6}{|c|}{ 'Brewster' (Ralo rojo) } \\
\hline Tolome & $44.36 \pm 5.4$ & $0.6 \pm 0.1$ & $19.0 \pm 0.7$ & $47.9 \pm 6.7$ & $39.0 \pm 3.9$ \\
\hline
\end{tabular}

${ }^{9}$ Media y desviación estándar de 40 frutos para cada huerta. "Letras iguales de una misma columna no son estadísticamente diferentes, (Tukey, 0.05). AT = acidez titulable; SST = sólidos solubles totales; DMS = diferencia mínima significativa.

$21.8 \%$. El peso de semilla fue de 9.7 a $15.8 \%$ del peso del fruto en 'Mauritius' y en 'Brewster' fue de $16.5 \%$ (Figura 2). Resultados similares fueron reportados por Holcroft y Mitcham (1996) en el cultivar 'Mauritius', donde el arilo representó de 70 a $75 \%$ del total, la cáscara de 14 a $16 \%$ y la semilla de 11 a $14 \%$ del peso del fruto. Por su parte, Martins et al. (2001) reportaron $71 \%$ de pulpa para el fruto de 'Brewster', ligeramente superior a los resultados de este estudio.

De estos valores, es importante destacar que los frutos del cultivar 'Mauritius' producidos en la huerta de Tuxpan, presentaron el mayor porcentaje de pulpa y los menores porcentajes en cáscara y semilla, en comparación con las otras huertas. Una posible explicación de esta diferencia es su ubicación geográfica a la mayor latitud norte (Cuadro 1), por lo que sus condiciones climáticas son más cercanas a las exigencias del cultivo de litchi, aunado a las características del suelo en esta huerta.

Por otro lado, entre los dos cultivares aquí evaluados se apreciaron importantes diferencias en los componentes del fruto, ya que 'Brewster' presentó mayor porcentaje de cáscara y semilla, y por consecuencia menor porcentaje de pulpa, en comparación con 'Mauritius'. Estas diferencias varietales tienen relación con las características propias del cultivar 'Brewster' que tiene cáscara más gruesa y semilla de mayor tamaño, pero también se asocia con las condiciones edafo-climáticas de la huerta Tolome, que no cumplen los requerimientos del cultivo señalados por Menzel (2002), lo que debió haber afectado al desarrollo del fruto.

\section{CONCLUSIONES}

Las diferencias edafo-climaticas y de manejo agronómico entre las huertas influyeron en la calidad de los frutos de litchi de los cultivares 'Mauritius' y 'Brewster'.

La huerta de Lechuguillas bajo un manejo orgánico fue la que presentó las mejores características de calidad del fruto en el cultivar 'Mauritius'. Las variables de calidad de fruto sobresalientes en esta huerta son mayor tamaño de fruto (35.1 $\mathrm{mm}$ de longitud y $21.2 \mathrm{~g}$ de peso), mayor peso de pulpa (16.8 g), baja acidez titulable ( $0.4 \%)$, alto contendio sólidos solubles totales $\left(19.3^{\circ} \mathrm{Brix}\right)$, y buen color de cáscara (33.7 Chroma).

\section{AGRADECIMIENTOS}

Al Consejo Nacional de Ciencia y Tecnología (CONACYT) por el financiamiento del proyecto clave 90870 y por la beca No. 231601 otorgada para estudios de maestría. A la Línea Prioritaria de Investigación "Agroecosistemas Sustentables" del Colegio de Postgraduados, por las facilidades otorgadas para hacer la investigación.

\section{BIBLIOGRAFÍA}

AOAC, Association of Official Analytical Chemists (2000) Official Methods of Analysis.16th ed. S. William (ed). Published by the Association of Official Analytical Chemists. Washington, D.C. USA. CD-Rom.

Aquino B. E. N., R. Corona V., A. Villegas C., I. Reyes S., N. Güemes V., A. D. Hernández F. and E. Mercado S. (2010) Effect of storage temperature and time on quality in minimally processed litchi fruit (Litchi chinensis Sonn.). Journal of Food Quality 33:299311.

Aular S., C. Ruggiero y J. Durigan (2002) Relación entre el color de la cáscara y las características del fruto y jugo de la parchita maracuyá. 


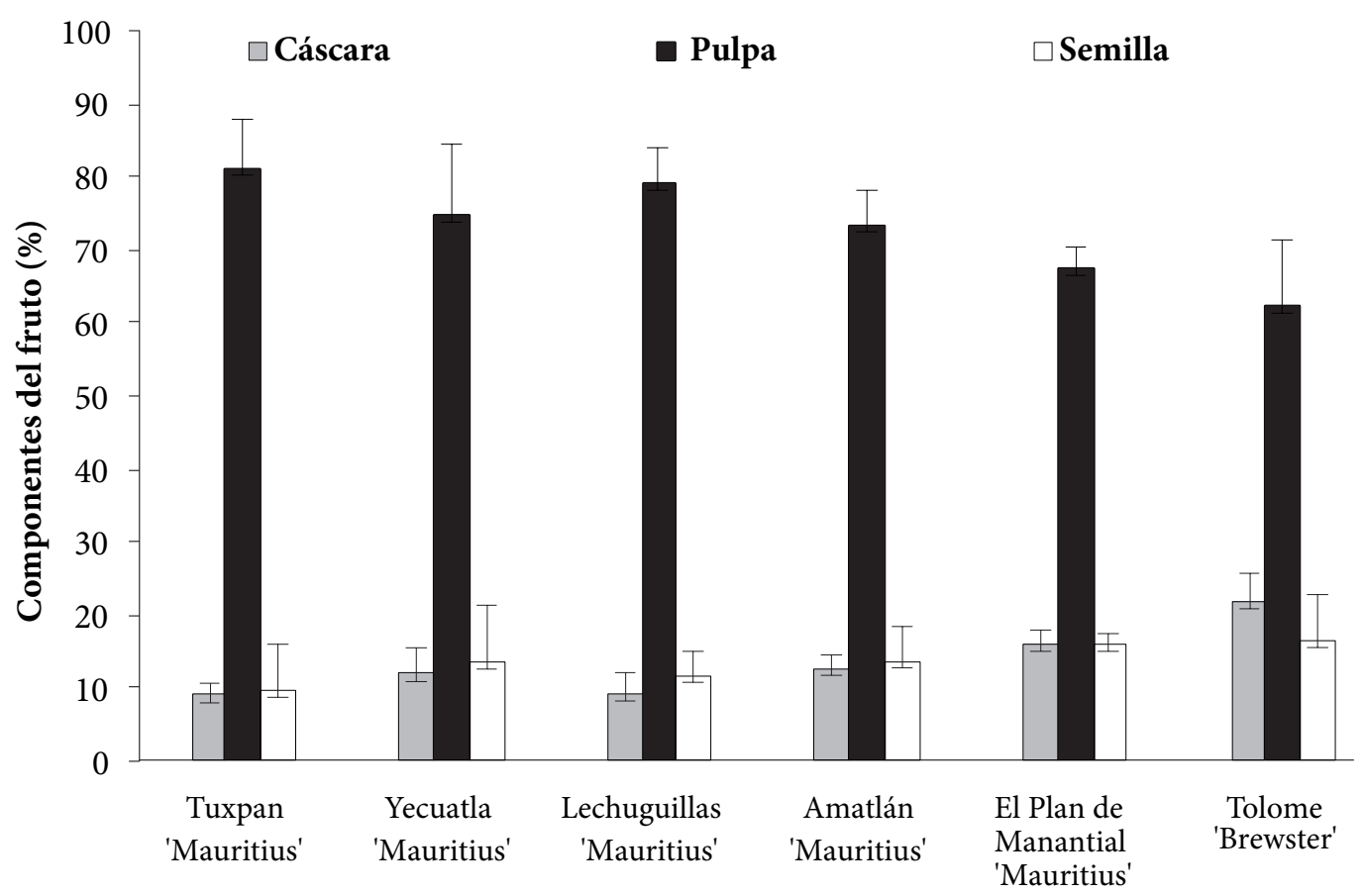

Figura 2. Componentes del fruto de litchi de los cultivares 'Mauritius' y 'Brewster' en seis localidades en el estado de Veracruz, México.

Bioagro 14:47-51.

Carvalho C. M. \& L. C. Ch. Salomão (2000) Cultura da Lichieira. Boletim de Extensão 43. Universidade Federal de Viçosa, ViçosaMG. 39 p.

Cronje R. B., D. Sivakumar, P. G. Mostert and L. Korsten (2009) Effect of different preharvest treatment regimes on fruit quality of litchi cultivar Mauritius. Journal of Plant Nutrition 32:19-29.

De la Garza N. J. A. (2004) El litchi: Alternativa para la diversificación frutícola en la Huasteca Potosina. Folleto técnico No. 117. INIFAP. Huichihuayan, San Luis Potosí, México. 40 p.

De Reuck K., D. Sivakumar and L. Korsten (2009) Integrated application of 1-methylcyclopropene and modified atmosphere packaging to improve quality retention of litchi cultivars during storage. Postharvest Biology Technology 52:71-77.

FAO/WHO, Food and Agriculture Organization of the United Nations/ World Health Organization (2011) Codex Alimentarius: Codex Standard for Litchi. Disponible en: http:/www.codealimentarius.net/download/standars/320/CXS196e.pdf. (Marzo 2010).

Galán Sauco V. y U. G. Menini (1987) El Litchi y su Cultivo. Estudio FAO Producción y Protección Vegetal 83. (FAO), Roma, Italia. 214 p.

Global Freigth Solutions-US Customs Compliance (2014) Disponible en: http://profreight.us/blog/2013/09/24/u-s-allows-australian-litchi-mango-imports/. (Febrero 2014).

Hajare S. N., S. Saxena, S. Kumar, S. Wadhawan, V. More, B. B. Mishra, M. Narayan P., S. Gautam and A. Sharma (2010) Quality profile of litchi (Litchi chinensis Sonn.) cultivars from India and effect of radiation processing. Radiation Physics and Chemistry 79:994-1004.

Holcroft D. M. and E. J. Mitcham (1996) Postharvest physiology and handling of litchi (Litchi chinensis Sonn.). Postharvest Biology Technology 9:265-281.

Jiang Y, L. Yao, A. Lichter and J. Li (2003) Postharvest biology and technology of fruit. Journal Food, Agriculture \& Environment 1:7681.

Kumar D., D. Shankar M., B. Chakraborty and P. Kumar (2011) Pericarp browning and quality management of litchi fruit by antioxidants and salicylic acid during ambient storage. Journal of Food Science and Technology 12:89-96.
López A. and P. Gómez (2004) Comparison of color index for tomato ripening. Horticultura Brasileira 22:534-537.

Martins A. B. G., D. C. Bastos e J. Scaloppi (2001) Lichieira (Litchi chinensis Sonn.). Jaboticabal-SP. Série Frutas Potenciais Sociedade. Brasileira de Fruticultura. 48 p.

McGuire R. G. (1992) Reporting of objective color measurements. Horticultural Science 27:1254-1255.

Menzel C. M. (2002) Overview of lychee production in the Asia-Pacific region. In: Lychee Production in the Asia-Pacific Region. M K. Papademetriou and F. J. Dent (eds.). Food and Agricultural Organization of the United Nations, Bangkok, Thailand. $128 \mathrm{p}$.

Mitra S. K. and P. K. Pathak (2010) Litchi production in the Asia-Pacific region. Acta Horticulturae 863:29-36.

Nacif S. R., A. A. Sartori P. and L. C. Chamhum (2001) Morphological and anatomical development of the litchi fruit (Litchi chinensis Sonn. cv. 'Brewster'). Fruits 56:225-233.

National Agricultural Marketing Council (2013) South Africa Fruit Trade Flow. Markets and Economic Research Centre. Issue 11 September. Avalaible at: http://www.namc.co.za/upload/Fruitflow-trade-No. 11-Sept-Edition.pdf. (Febrero 2014).

Neog M. and L. Saikia (2001) Compositional changes in litchi (Litchi chinensis Sonn.) during fruit development. Journal Agricultural Science Society 14:208-212.

Pesis E., O. Diver, O. Feygenberg, A. R. Ben, M Ackerman and A. Lichter (2002) Production of acetaldehyde and ethanol during maturation and modified atmosphere storage of litchi fruit Postharvest Biology and Technology 26:157-165.

Rinderman R. S. y C. M. Gómez (2001) El Litchi. 2a ed. Ed. Mundi-Prensa. México. $144 \mathrm{p}$.

Sivakumar D. and L. Korsten (2006) Influence of modified atmosphere packaging and postharvest treatments on quality retention of litchi cv. 'Mauritius'. Postharvest Biology and Technology $41: 135-142$

SAS Institute (2004) SAS ${ }^{\circledR} 9.1$ SQL Procedure User's Guide. Cary, NC, USA.

SIAP, Servicio de Información Agroalimentaria y Pesquera (2014) Anuario estadístico de la producción agrícola. Secretaría de Agricultura, Ganadería, Desarrollo Rural, Pesca y Alimentación. México. Disponible en: http://www.siap.sagarpa.gob.mx. 
(Febrero 2014).

Soto E. M. (1986) Localidades y Climas del estado de Veracruz. Ed. Herb. Instituto Nacional de Investigaciones sobre Recursos Bióticos. Xalapa, Veracruz, México.137 p.

Valle-Guadarrama S., A. Alonso-Campos y I. Alia-Tecajal (2008) Atmósferas con bajo $\mathrm{O}_{2}$ y alto $\mathrm{CO}_{2}$ para la conservación de frutos de litchi. Revista Fitotecnia Mexicana 31:157-164.
Wyszecki G. and W. S. Stiles (1982) Color Science Concepts and Methods, Quantitative Data and Formulae. John Wiley \&Sons, Inc. Canada. 935 p.

Zhou B., H. Chen, X. Huang, N. Li, Z. Hu, Z. Gao and Y. Lu (2008) Rudimentary leaf abortion with the development of panicle in litchi: changes in ultraestructure, antioxidant enzymes and phytohormones. Scientia Horticulturae 117:288-292. 